\title{
New optical spectra and general discussion on the nature of ULXs
}

\author{
H. Arp ${ }^{1}$, C. M. Gutiérrez ${ }^{2}$, and M. López-Corredoira ${ }^{3}$ \\ ${ }^{1}$ Max-Planck-Institut für Astrophysik, Karl Schwarzschild-Str.1, Postfach 1317, 85741 Garching, Germany \\ 2 Instituto de Astrofísica de Canarias, 38200 La Laguna, Tenerife, Spain \\ e-mail: cgc@ll.iac.es \\ 3 Astronomisches Institut der Universität Basel, Venusstrass 7, 4102 Binningen, Switzerland \\ e-mail: martinlc@astro.unibas.ch
}

Received 16 September 2003 / Accepted 29 January 2004

\begin{abstract}
We present optical spectroscopic observations of three Ultra Luminous X-ray sources (ULXs). Two of them are very close to the active galaxy NGC 720 and the other is near NGC 1073. The two around NGC 720 turn out to be quasars at $z=2.216$ and $z=0.959$, the one near NGC 1073 seems to be associated to an HII region at the redshift of NGC 1073. We concentrate our analysis on the two quasars and analyze them in conjunction with a set of 22 additional X-ray sources close to nearby galaxies which also fit the criteria of ULXs and which also have been identified as quasars of medium to high redshift. This sample shows an unusually large fraction of rare BL Lac type objects. The high redshifts of these ULXs and their close proximity to their low redshift, supposedly parent galaxies is a surprising result in the light of standard models. We describe the main properties of each of these objects and their parent galaxy, and briefly discuss possible interpretations.
\end{abstract}

Key words. galaxies: active - galaxies: quasars: general - X-rays: galaxies

\section{Introduction}

Numerous X-ray sources have been found in the central regions of nearby galaxies such as M 31 and succesfully attributed to the presence of X-ray binary stars (e.g. Supper et al. 1997). A number of objects with much greater luminosities in the range $L_{\mathrm{X}}=10^{39}$ to $10^{41} \mathrm{erg} \mathrm{s}^{-1}$ have also been found in nearby galaxies. In that case they are designated "Ultra Luminous X-ray sources" (ULXs). It has been proposed that they are exceptionally massive black hole binaries. If, however, at the distance of the parent galaxy a source has an X-ray luminosity exceeding about $L_{\mathrm{X}}=10^{38.5} \mathrm{erg} \mathrm{s}^{-1}$, it needs to be more massive than a normal stable star (Pakull \& Mirioni 2002 quote an Eddington limit as the upper bound on the luminosity of an accreting star as $L_{\mathrm{X}}=10^{38.1} \mathrm{M} / \mathrm{M}_{\odot} \mathrm{erg} \mathrm{s}^{-1}$ and Foschini et al. (2002a) quote $L_{\mathrm{X}}=10^{38.3} \mathrm{erg} \mathrm{s}^{-1}$ as the most luminous $\mathrm{X}$-ray binaries in $\mathrm{M}$ 31). Other explanations have been proposed in terms of black holes in globular clusters (Angelini et al. 2001), or associations with HII regions (Roberts et al. 2003; Pakull \& Mirioni 2002; Wang 2002; Gao et al. 2003). Alternative explanations have been proposed in terms of nonisotropic emission of the radiation (King et al. 2001; Körding et al. 2002). But, as discussed here, an important fraction of the ULXs which have been so far spectroscopically observed have higher redshift than the parent galaxy. So, currently the nature

Send offprint requests to: $\mathrm{H}$. Arp,

e-mail: arp@mpa-garching.mpg.de of these objects remains unexplained, and it is necessary to rexamine the assumption that they are physically associated with the galaxies in which they are found.

The most extensive catalog of ULXs is the one presented by Colbert \& Ptak 2002 (CP02 hereafter) in which 87 ULXs in the near field of $54 \mathrm{RC} 3$ galaxies with $c z \leq 5000 \mathrm{~km} \mathrm{~s}^{-1}$ are compiled. However, these authors have filtered out known quasars and $\mathrm{X}$-ray supernova. We think this is unjustified until the true nature of the ULXs become clear. In this paper we do not remove such objects, use only the $L_{\mathrm{X}}$ criterion to define ULX objects and change slightly the range in X-luminosity to define ULXs, $L_{\mathrm{X}} \geq 10^{38.5} \mathrm{erg} \mathrm{s}^{-1}$.

To disentangle the nature of these objects it is necessary to find their possible optical counterparts and identify their characteristic optical spectral features. However, this is not an easy task (see Foschini et al. 2002b) due to the uncertainties in the $\mathrm{X}$-ray coordinates and the optical faintness of many of these objects. In this paper we present the optical identification and spectroscopic observations of three ULXs, two of them (IXO1 and IXO2 according to the notation used in CP02) in the field of the galaxy NGC 720 and one (IXO5) around NGC 1073.

\section{Observations and data reduction}

We found probable optical counterparts in the Digital Sky Survey (DSS) plates for objects IXO1 and 2 around NGC 720 (the existence of the optical counterparts of these 


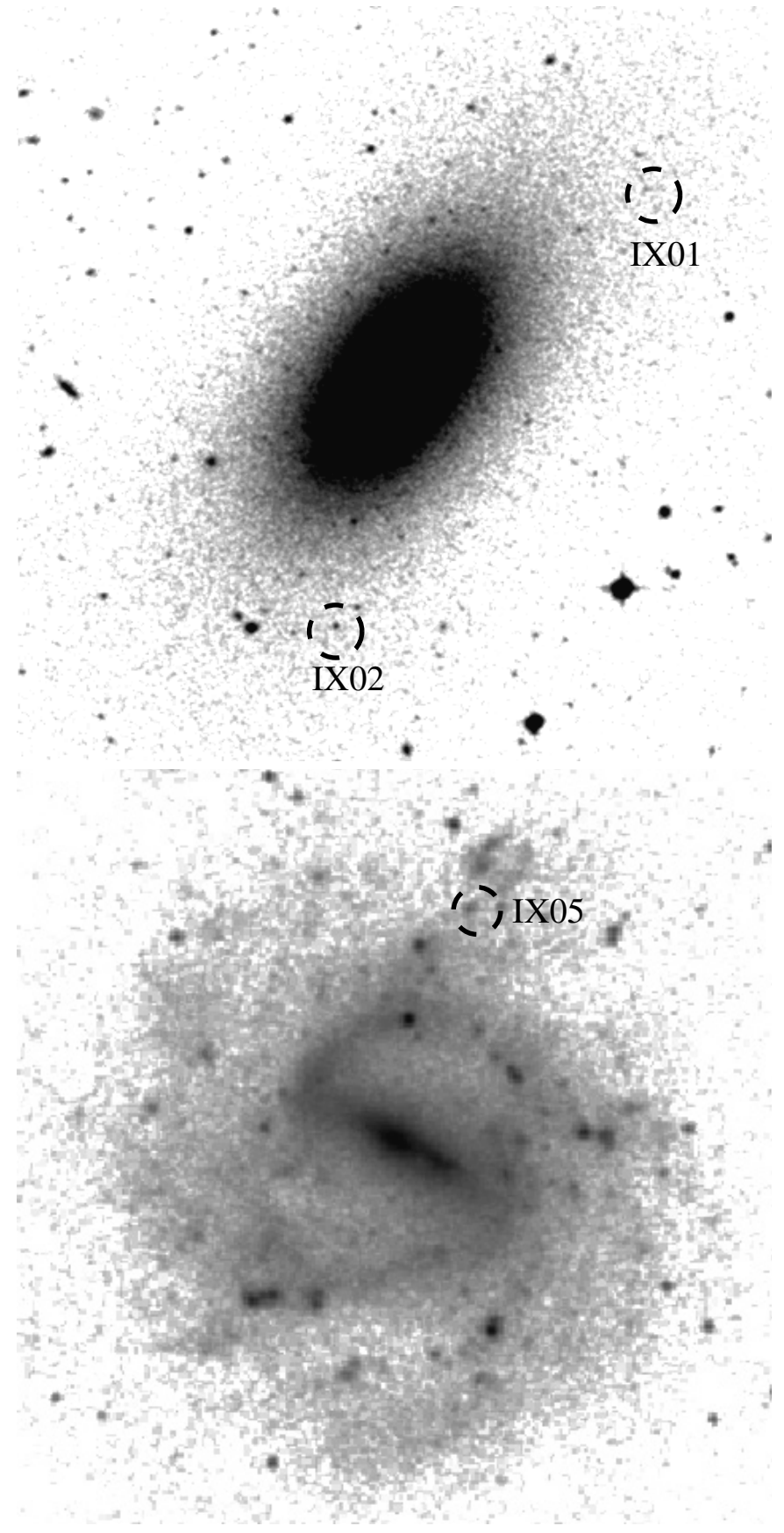

Fig. 1. DSS plate centred on NGC 720 and NGC 1073 with field of views of $5^{\prime} \times 5^{\prime}$. Indicated are the positions of the optical counterparts of the objects IXO1, IXO2 and IXO5. See main text for the details.

two objects was pointed out by CP02) and IXO5 around NGC 1073. Figure 1 shows DSS plates centered on NGC 720 and NGC 1073, and the position of the possible optical counterparts of the IXO objects. We obtained long-slit spectroscopy of these three objects with the WHT ${ }^{1}$ on 28-29 Dec. 2002. We used the red arm of the ISIS spectrograph with the grism R158R. The slitwidth was 1 arcsec. This provides a

\footnotetext{
${ }^{1}$ The William Herschel Telescope (WHT) is operated by the Isaac Newton Group and the IAC in Spain's Roque de los Muchachos Observatory.
}

sampling of $1.62 \AA$ pixel $^{-1}$ and a resolution of $8 \AA$. For each object we took a single image with a exposure time of $1800 \mathrm{~s}^{2}$ The data were reduced using IRAF ${ }^{3}$. Further details of the observing conditions and data reduction can be found in López-Corredoira \& Gutiérrez (2004).

The extracted spectra of the three objects are presented in Fig. 2. All of them are emission line objects. It is evident from the optical spectra that objects IXO1 and IXO2 around NGC 720 are normal appearing quasars. From the main spectral features indicated in Fig. 2, we obtain redshifts of $z=$ $2.216 \pm 0.004$ and $z=0.959 \pm 0.004$ for IXO1 and IXO2 respectively. From the DSS plates, and assuming flat spectra, we have estimated $V$ magnitudes of 20.6 and 19.2 for the optical counterparts of IXO1 and IXO2 respectively. Given the paucity of quasars and the fact these have strong X-ray emission, we think the identification of these objects is unequivocal.

The optical object that we have associated with IXO5 has strong narrow emission lines. From the position of the main lines we measure a redshift of $z=0.0037 \pm 0.0002$ or in velocity $1200 \mathrm{~km} \mathrm{~s}^{-1}$. According to the ratio of $\mathrm{OIII}(5007) / \mathrm{H} \beta$ and $\mathrm{H} \alpha / \mathrm{NII}(6584)$, this object should be a HII region. Its redshift is similar to that quoted in NED for the galaxy NGC 1073 and it is in a region close to one of the spiral arms. The abundance of HII regions in the spiral arms makes the identification of the optical counterpart of the X-ray emission not as clear as for the objects IXO1 and IXO2 (furthermore there are a much fainter optical sources near this HII region as can be seen in Fig. 1). In any case, if the identification is correct, IXO5 would be associated with ionized gas, with the X-ray photons of the ULX as the possible source of ionization. Gao et al. (2003) have found more than a dozen of ULXs apparently associated to the outer, active star-forming ring in the Cartwheel ring galaxy.

\section{A compilation of ULXs identified as quasars}

In Table 1 we present a compilation from the literature of objects which fit the definition of ULXs and have been spectroscopically identified. Three of the objects (the ones denoted as IXO1, IXO2 and IXO43) are listed as ULXs in CP02. The rest of the objects, although they fit the observational criteria of classification as ULXs, were not included in the above catalog apparently because they were known quasars with higher redshift than the parent galaxy. The parameters quoted in this table are:

Col. 1: the designation ULX (or IXO in the CP02 catalog) is based on a $\log L_{\mathrm{X}} \geq 38.5 \mathrm{erg} \mathrm{s}^{-1}$ as estimated in Col. 5;

Cols. 2 and 3: the Right Ascension and Declination of each object (epoch 2000);

Col. 4: the X-ray counts/ks measured whenever possible from the ROSAT HRI detector in order to match as closely

\footnotetext{
${ }^{2}$ One additional exposure of worse quality was obtained for IXO1 and IXO2 respectively. Although not considered here, those spectra are perfectly consistent with the ones presented in this paper.

${ }^{3}$ IRAF is the Image Reduction and Analysis Facility, written and supported by the IRAF programming group at the National Optical Astronomy Observatories (NOAO) in Tucson, Arizona.
} 

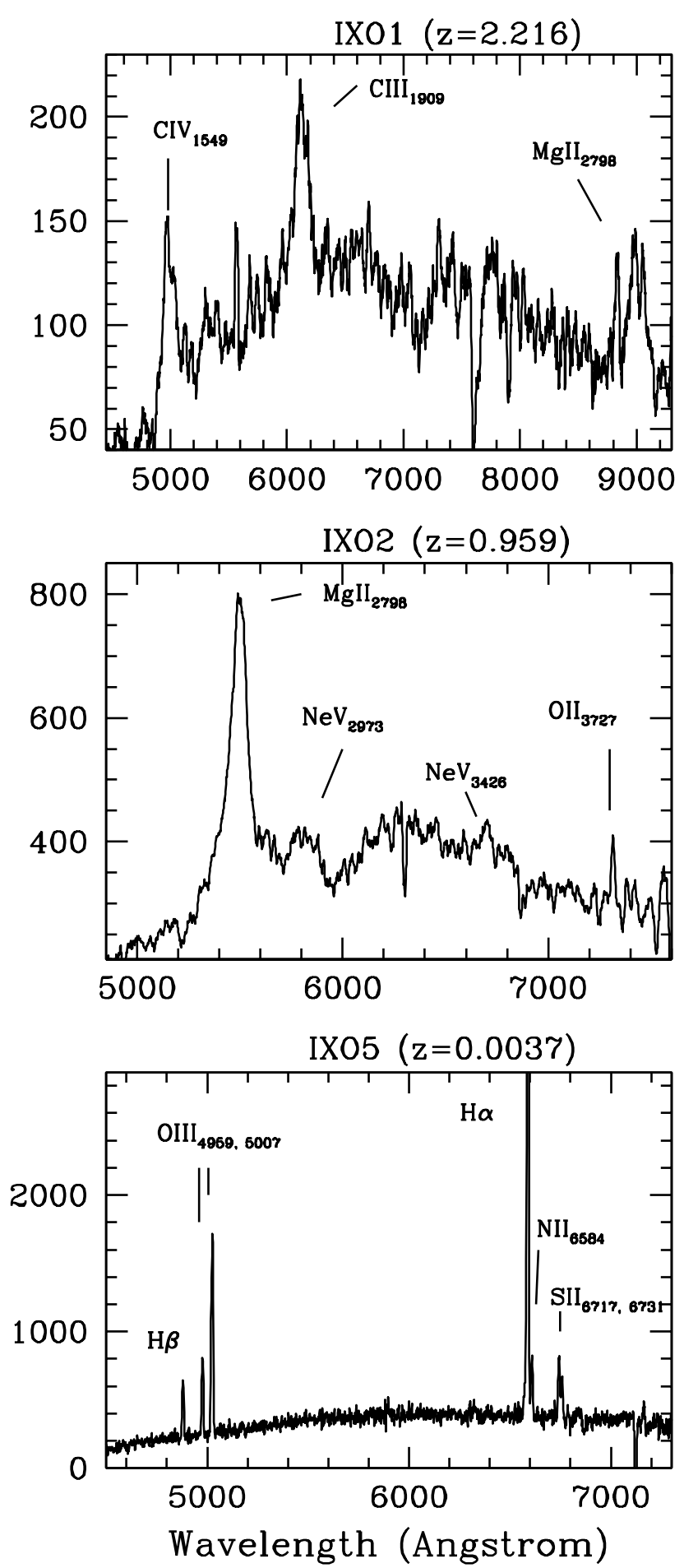

Fig. 2. Spectra measured with the William Herschel telescope of the ULXs called IX01, IX02, IX05 in the Colbert and Ptak Catalog (2002). The vertical axis is the instrumental flux in arbitrary units. The main emission lines and the redshifts are indicated.

as possible the $\mathrm{CP} 02$ flux criteria. In a few cases outside the HRI field they were estimated from PSPC observations;

Col. 5: the X-ray luminosity $\left(\log L_{X}\right)$ if the ULXs are at the same distance as the associated low-redshift galaxies (see discussion below). If the ULXs were not at the same distance, the real luminosities would have to be multiplied roughly by $\left(\frac{d_{\mathrm{ULX}}}{d_{\text {galaxy }}}\right)^{2}$. Except for the IXOs, most of these values have been calculated by the relation between the HRI counts/ks and the CP02 luminosities.

Most of the galaxies have redshift $0.003 \leq z \leq 0.005$ where the contribution to this redshift from their peculiar velocities could be significant. Therefore, except for NGC 720, NGC 4168 and NGC 4258 the distances used for the ULXs in Table 1 are the distance to the associated Local Supercluster, 15.3 Mpc. (Hubble Key Project; Freedman et al. 2001). The flux calculations vary somewhat from hard to soft bands and the raw counts are subject to variability in most sources. We have only attempted to get the calculated luminosities approximately on the scale of other catalogs;

Col. 6: the redshift $\left(z_{Q}\right)$ measured for the object which has been optically identified with the X-ray source;

Col. 7: the apparent magnitude is in the $V$ band but in a few cases in other bands as individually noted in Véron-Cetti \& Véron (2003);

Cols. 8 and 9: the name and redshift $\left(z_{G}\right)$ of the host or parent galaxy;

Col. 10: the radial distance ( $r$ ), in arcmin, from the center of the galaxy to the position of the ULX. This usually is about 4-5 arcmin for IXO identifications but we have included a few here with slightly larger acceptance distances as individually noted.

Col. 11: the absolute optical magnitude (see above note in Col. 7);

Col. 12: a note to indicate BL Lac type objects;

Col. 13: references.

\subsection{Comments on individual objects}

- NGC 720: these two IXO's lie very close to a bright $\left(B_{T}=\right.$ $11.15 \mathrm{mag}) \mathrm{E}$ galaxy. The galaxy is a very strong ROSAT $\mathrm{X}$-ray source which emits $148 \mathrm{cts} / \mathrm{ks}$. Moreover, there is a strong, "twisted", X-ray jet/filament which curves southward as it emerges from the nucleus (Buote \& Canizares 1996). In addition to the two ULXs, X-ray quasar candidates of similar apparent magnitude are conspicuous at larger radial separations from NGC 720 (see "Catalogue of Discordant Redshift Associations", Arp 2003).

- NGC 1073: in the CP02 catalog there are two X-ray sources in this galaxy which are at about the same distance from the nucleus and much brighter in X-rays than the source which they designated IXO5 (this is associated to a HII region if our identification was correct, as said). These two were not include in the IXO CP02 catalog because it was known that they were quasars (Arp \& Sulentic 1979; Burbidge et al. 1979). But clearly these fit the definition of ULXs and are listed in Table 1. Also included in Table 1 is the third known quasar in NGC 1073 which has $\log L_{\mathrm{X}}=38.7 \mathrm{erg} \mathrm{s}^{-1}$ only slightly above the general criterion for ULXs. The strong $\mathrm{X}$-ray quasar with $z=1.411$ is also a strong radio source. Since high redshift compact objects which are strong in both X-ray and radio are generally now regarded as BL Lac type objects it is noted in the last column of Table 1. 
Table 1. Properties of objects analyzed in this paper.

\begin{tabular}{|c|c|c|c|c|c|c|c|c|c|c|c|c|}
\hline ID & $\begin{array}{c}\text { RA } \\
\text { (hh mm ss) }\end{array}$ & $\begin{array}{c}\text { Dec } \\
(\mathrm{deg} \mathrm{mm} \mathrm{ss})\end{array}$ & $\mathrm{cts} / \mathrm{ks}$ & $\begin{array}{c}\log \left(L_{X}\right) \\
\operatorname{erg~s}^{-1}\end{array}$ & $z_{Q}$ & mag & Host galaxy & $Z z_{G}$ & $\begin{array}{c}r \\
(\operatorname{arcmin})\end{array}$ & $M_{\text {abs }}$ & Type & References \\
\hline IXO1 & 015249.7 & -134211 & 0.50 & 39.1 & 2.216 & 20.6 & NGC 720 & 0.006 & 3.4 & -11.3 & $\ldots$ & 1 \\
\hline IXO2 & 015302.9 & -134653 & 1.77 & 39.7 & 0.959 & 19.2 & & & 2.7 & -12.7 & $\ldots$ & 1 \\
\hline ULX & 024339.4 & 012109 & 1.2 & 39.2 & 1.411 & 20.0 & NGC 1073 & 0.004 & 1.4 & -10.9 & BL Lac & 2 \\
\hline ULX & 024339.5 & 012220 & 0.4 & 38.7 & 1.945 & 19.8 & & & 1.8 & -11.1 & $\ldots$ & 2 \\
\hline ULX & 024333.3 & 012136 & 3.0 & 39.6 & 0.599 & 18.8 & & & 2.1 & -12.1 & $\ldots$ & 2 \\
\hline \multirow[t]{2}{*}{ ULX } & 033407.1 & -360400 & 3.6 & 39.8 & 0.904 & 19.7 & NGC 1365 & 0.005 & 7.7 & -11.2 & $\ldots$ & 3 \\
\hline & 033312.2 & -361948 & 38.8 & 40.9 & 0.308 & 18.0 & & & 12.4 & -12.9 & BL Lac & 3 \\
\hline \multirow[t]{7}{*}{ ULX } & 112014.7 & 133228 & 0.83 & 38.8 & 0.995 & 20.1 & NGC 3628 & 0.003 & 3.0 & -10.8 & $\ldots$ & 4 \\
\hline & 112011.9 & 133123 & 1.39 & 39.0 & 2.15 & 19.5 & & & 4.2 & -11.4 & $\ldots$ & 4 \\
\hline & 112006.2 & 134024 & 2.50 & 39.3 & 0.981 & 19.2 & & & 5.5 & -11.7 & $\ldots$ & 4 \\
\hline & 112039.9 & 133620 & 0.83 & 38.8 & 0.408 & 19.6 & & & 5.6 & -11.3 & $\ldots$ & 4 \\
\hline & 112041.6 & 133551 & 0.83 & 38.8 & 2.43 & 19.9 & & & 6.0 & -11.0 & $\ldots$ & 4 \\
\hline & 111946.9 & 133759 & 1.67 & 39.1 & 2.06 & 19.6 & & & 7.9 & -11.3 & $\ldots$ & 4 \\
\hline & 112106.1 & 133825 & 6.11 & 39.6 & 1.94 & 18.3 & & & 12.3 & -12.6 & $\ldots$ & 4 \\
\hline \multirow[t]{2}{*}{ IXO43 } & 121007.9 & 392312 & 0.88 & 39.3 & 0.239 & 18.4 & NGC 4151 & 0.003 & 5.0 & -12.5 & $\ldots$ & 5 \\
\hline & 121026.7 & 392909 & 63-106 & $40.6-40.9$ & 0.615 & 20.3 & & & 4.9 & -10.6 & BL Lac & 5 \\
\hline ULX & 121214.6 & 131248 & 4.0 & 39.4 & 0.217 & 18.7 & NGC 4168 & 0.0076 & 0.75 & -13.9 & $\ldots$ & 6 \\
\hline ULX & 121509.2 & 330955 & 38.4 & 40.7 & 0.614 & 17.5 & NGC 4203 & 0.004 & 2.1 & -13.4 & BL Lac & 7 \\
\hline ULX & 121808.3 & +471615 & 5.3 & 38.8 & 0.398 & 20.4 & NGC 4258 & 0.0015 & 8.6 & -8.6 & $\ldots$ & 8 \\
\hline ULX & 121952.3 & +472058 & 1.2 & 38.4 & 0.653 & 19.9 & & & 9.7 & -9.1 & $\ldots$ & 8 \\
\hline ULX & 121923.1 & +470939 & 3.9 & 38.7 & 0.52 & 17.0 & & & 9.7 & -9.1 & $\ldots$ & 9 \\
\hline ULX & 122144.0 & 751838 & 255 & 41.5 & 0.071 & 15.2 & NGC 4319 & 0.005 & 0.7 & -15.7 & BL Lac & 10 \\
\hline ULX & 122512.0 & 122153 & 5.6 & 39.8 & 1.25 & 18.5 & NGC 4374 & 0.004 & 2.4 & -12.4 & $\ldots$ & 11 \\
\hline ULX & 124825.9 & 083020 & $\ldots$ & 39.5 & 0.43 & 20.5 & NGC 4698 & 0.003 & 1.2 & -10.5 & BL Lac & 12 \\
\hline
\end{tabular}

References: 1 This work; 2 Arp, H., \& Sulentic, J. 1979, ApJ, 229, 496; 3 F., La Franca, F., Fiore, F., Vignali, C., et al. 2002, ApJ, 570, L100; 4 Arp, H., Burbidge, E. M., Chu, Y., Flesch, E., Patat, F., \& Rupprecht, G. 2002, A\&A, 391, 833; 5 Arp, H. 1977 ApJ, 218, 70; 6 Masetti, N., et al. 2003, ApJ, 406, L27; 7 Knezek, P. M., \& Bregman, J. N. 1998, AJ, 115, 1737; 8 Burbidge, E. M. 1995, A\&A, 298, L1; 9 Mirioni, L. 2003 Ph.D. Thesis, University of Strasbourgh; 10 Stocke, J. T., et al. 1991, ApJS, 76, 813; 11 Burbidge, G., Hewitt, A., Narlikar, J. V., \& Das Gupta, P. 1990, ApJS, 74, 675; 12 Foschini, L., Ho, L., Masetti, N., et al. 2002, A\&A, 396, 787.

- NGC 1365: this is a well known Seyfert Galaxy in the Fornax Cluster. About 12 arcmin to the SE in the direction of its extended spiral arm lies a very strong X-ray BL Lac (Arp 1998, p. 49). The distance to the Fornax Super Cluster is about the same as to the Virgo Super Cluster in the opposite direction in the sky, or about 15 Mpc (or see Arp 2002 for a Cepheid distance of $18 \mathrm{Mpc}$ ). With this distance to NGC 1365, the BL Lac object comes within the acceptance radius of the IXO Catalog and is included in Table 1.

Closer in radial separation on the other side of NGC 1365 is a strong X-ray source with $z=0.904$ (La Franca et al. 2002). 
- NGC 3628: this starburst/low level AGN exhibits extensive outgassed plumes of neutral hydrogen and is apparently ejecting X-ray material along its minor axis. Point X-ray sources might be associated with this ejected material and a number have already turned out to be quasars (Arp et al. 2002). We lists 7 of these quasars which have $\log L_{X}$ between 38.8 and $39.6 \mathrm{erg} \mathrm{s}^{-1}$. Column 10 shows that six of these quasars fall within the same radial distances as most of the ULXs in this table. The seventh, which has the strongest, $\log L_{X}=39.6 \mathrm{erg} \mathrm{s}^{-1}$, falls at a greater distance, very similar to the radial distance of the bright BL Lac from NGC 1365.

- NGC 4151: there is a powerful and variable X-ray source only $4.9 \operatorname{arcmin} N$ of this active Seyfert galaxy. It is also a strong radio source (de Ruiter et al. 1977) but, surprisingly, quite faint optically. Later it was shown that there was an X-ray filament apparently linking this $z=0.615$ BL Lac quasar to the nucleus of NGC 4151 (Arp 1997, Fig. 17). The object should have been included in the CP02 cata$\log$ but they centered their field to the SW of NGC 4151, just leaving it out of their frame. As a result this important BL Lac object was not listed as an IXO.

A very interesting object SW of NGC 4151 was, however, listed as IXO 43. It turns out to be a galaxy on the end of a chain of seven or eight galaxies (Arp 1977). This end galaxy on the chain has an emission spectrum of about $z=0.239$ and is apparently the identification of the Ultra Luminous X-ray Source (IXO 43). Three other members of the chain have redshifts near $z=0.160$ and there are indications of absorption redshifts of $z=0.04$ to 0.06 .

- NGC 4168: a faint AGN galaxy at redshift $z=0.217$ is the ULX close to this Seyfert 1 galaxy which has $z=0.0076$ (Masetti et al. 2003). It is similar to the objects found in the filament from the Seyfert NGC 7603 (López-Corredoira \& Gutiérrez 2002, 2004).

- NGC 4203: a remarkably similar object to the $z=$ 0.615 BL Lac object near NGC 4151, is the strong X-ray source only 2.1 arcmin from NGC 4203. It has a redshift of $z=0.614$. This is another source which was not included in the $\mathrm{CP} 02$ catalog due to previously known high redshift. NGC 4203 is a liner type AGN with broad Balmer lines. It is also a strong X-ray source and an IRAS infrared source. IXO45 is just W of the BL Lac, Tonanzintla 1480, and optically identified with a blue quasar candidate. Chandra snapshots of this field show the galaxy X-ray emission and the strong emission of the adjacent ULXs (Terashima \& Wilson 2003).

- NGC 4258 is a Seyfert II galaxy with "anomalous" spiral arms. The distance used to compute the X-ray emission is $6.4 \mathrm{Mpc}$. This is a very interesting case in which three of the X-ray sources listed by Pietsch et al. (1994) have result quasars at higher redshift than the parent galaxy. The angular distance of these three objects with respect to NGC 4258 is very similar, with two of them (the objects with redshifts 0.398 add 0.653 lying nearly along the minor axis of NGC 4258. More details about these sources can be found in Pietsch et al. (1994); Burbidge (1995); Vogler \& Pietsch (1999) and Mirioni (2003).
- NGC 4319: this is an unusually disrupted spiral with a well known quasar/AGN, Markarian 205, only 40 arcsec south of its nucleus. As Table 1 shows this ULX is exceptionally strong in X-rays, the strongest in the table at $\log L_{X}=41.5 \mathrm{erg} \mathrm{s}^{-1}$, if it were at the redshift distance of NGC 4319, and much larger luminosity if the redshift were cosmological.

- NGC 4374: this is a large radio galaxy. The close X-ray source has long been known as a quasar of $z=1.25$. Again, this was not included in the $\mathrm{CP} 02$ catalog because its known high redshift.

- NGC 4698: the central galaxy here is a bright nearby Seyfert 2 galaxy. Foschini et al. (2002c) identified a ULX within only 73 arcsec of the nucleus of the galaxy. If it was an object at the same distance as the galaxy it would have a luminosity of $\log L_{X}=39.5 \mathrm{erg} \mathrm{s}^{-1}$. This ULX, however, turned out to be a quasar of $z=0.43$. In fact it turned out to be a pure absorption line BL Lac quasar which is a very rare type of object. Its apparent magnitude was quite red, $B=21.3$ and $R=19.6 \mathrm{mag}$. In order to get it roughly on the $V$ system we take a mean of these and get an absolute magnitude of $M_{\mathrm{abs}}=-10.4 \mathrm{mag}$, corresponding roughly to the lower limit of the optical luminosities of the ULX/quasars in Table 1.

Note: It is perhaps significant that almost exactly on a line through the nucleus of NGC 4698, extended beyond the BL Lac, is another, stronger X-ray source, 1RXS J124828.2+083103. It has a redshift of $z=0.120$. Extended further beyond that is a line of fainter galaxies of unknown redshift (Forschini et al. 2002c). It is very similar to the lines of galaxies extending from active low redshift galaxies that were found in (Arp 2001).

\section{Discussion}

Data available so far and compiled in Table 1 indicates that at least an important fraction of the ULXs are quasars. It is interesting to consider if some observational bias is present in the detection of the optical counterpart of ULXs. According to Bradt \& McClintock (1983) and Maccacaro et al. (1988) for AGNs the X-ray to visual flux ratio is $\sim 10-50$, this range is $\sim 0.1-10.0$ for high mass $\mathrm{X}$-ray binaries, and $\sim 100-1000$ for low mass binaries. Then, comparing the high mass X-ray binaries with AGNs, the observing bias would be favour the detection of binaries. This is particularly clear for the three objects detected around NGC 4258. Their optical luminosities are in the ramge of luminous stars and therefore we should have detected high mass X-ray binaries if they were there.

There is one respect in which the ULX quasars are different from the general run of quasars. Out of the 11 galaxies listed in Table 1, six have strong BL Lac objects associated with them as ULXs. A further case was recently discovered: in a recent Chandra investigation of the multiple interacting nuclei of NGC 4410 a possible population of ULX sources were investigated. Nowak et al. (2003) reported: "one of these sources is clearly associated with a radio point source, whereas another source (in fact the brightest in the system), may also be associated with radio emission". 


\subsection{Excess of $X$-ray quasars around active galaxies}

A first observation is that almost every one of the galaxies which have associated ULXs in Table 1 are Seyfert, starburst, radio or AGN active galaxies. One might argue that these are selectively the most X-ray observed class of galaxies, nevertheless it is striking that there is not even one, normal quiescent galaxy among the group. A systematic study of the kinds of galaxies most associated with quasars and ULXs would be useful. Apparently, this is in disagreement with the paper by Humphrey et al. (2003) which states that they found, with Chandra, 22 ULXs in a sample of 13 normal galaxies. However, the 7 galaxies from which those 22 ULXs actually come, include a Liner/AGN, a Seyfert, an X-ray cluster, an interacting radio X-ray pair (Arp 281) and a strong radio ejecting galaxy in the center of the Fornax Cluster (NGC 1399). For their five least active galaxies only one candidate ULX was found. So, it seems that in fact the active galaxies do preferentially produce the ULXs.

In their study of X-ray sources in fields around bright Seyfert galaxies, Radecke (1997) and Arp (1997) showed that there was an overdensity of bright, point X-ray sources compared to control fields at a significance level of $7.5 \sigma$. According to the present paper, these X-ray sources consistently turn out to be mostly quasars of a normal range in redshift. The radii from the central galaxy within which these X-ray quasars were found was roughly 5 to 50 arcmin (from the outer edges of the galaxies to the field limit of the PSPC, ROSAT detector). In the present case of the ULXs, the majority are found within about 5 arcmin of the central galaxy. Roughly speaking, there is a difference of about 100 in the areas concerned so that the chance of finding unrelated background sources is roughly $1 / 100$ of the probability in the larger area in which the $7.5 \sigma$ association was found. When some ULXs turned out to be much higher redshift, each of these was relegated, on an individual basis, to be a background source. Now, however, we have more than 20 cases, all turning out to be quasars but not one with a binary stellar spectrum.

Of the 24 Seyferts investigated in Radecke (1997) and Arp (1997) only four had BL Lac's associated. But it should be noted that those BL Lac's were the most significant associations with the surveyed active galaxies. Just by themselves the chance of finding those four was $2 \times 10^{-9}$. Here, in Table 1, we see two of those previous BL Lacs (with NGC 1365 and NGC 4151) but in addition, four new ones, four of which are extremely close, between 2.1 and 1.2 arcmin of the host galaxy so the probability to have them as background sources is much lower. The question then is: why would BL Lacs be more closely associated than ordinary quasars with Seyfert, starburst, radio or AGN galaxies?

\subsection{Very bright, off nuclear, Chandra ULXs}

Active galaxies like NGC 3628, Arp 220 and M 82 are now known to have very bright, ULX sources very close to their nuclei. Because these are generally even more X-ray luminous than the ones discussed here it would be especially important to attempt optical identifications with high resolution telescopes. For instance in NGC 3628 a source $\log L_{X}=$ $40 \mathrm{erg} \mathrm{s}^{-1}$ only 20 arcsec from the nucleus is reported as violently variable (Strickland et al. 2001). Arp 220 has a source $\log L_{\mathrm{X}}=40.6 \mathrm{erg} \mathrm{s}^{-1}$ only 7 arcsec off nucleus (Clements et al. 2002). NGC 7319, the Seyfert Galaxy in Stephan's Quintet has a ULX only 8 arcsec S of its nucleus. (Trinchieri et al. 2003); it is identified with a stellar appearing object at $B=21.78 \mathrm{mag}$ (Galianni \& Arp in preparation). M 82 has its most luminous X-ray source variable between $\log L_{\mathrm{X}} \sim 40$ to $41 \mathrm{erg} \mathrm{s}^{-1}$ (Matsumoto et al. 2001) lying within 8 arcsec of the M 82 nucleus, but no optical identifications have been made so it can only be surmised that it would turn out to be like, or a precursor of, the ULXs for which we have spectra in the present paper.

\subsection{Possible interpretation}

Arp $(1987,1998)$ suggested that quasars emerge from the nuclei of galaxies. In that model the quasars or proto quasars must pass out from the nucleus and out through the body and immediate environments of the galaxy before arriving at the distances where they have been shown to be physically associated. In this case they must pass through the region in which the present ULXs have been identified. It was therefore predicted that we should observe quasar-like objects in just the region in which observers are reporting ULXs (see Burbidge et al. 2003). Seyfert, starburst, radio or AGN active galaxies would be the parent ejecting galaxies. Rare cases such as the ULX in NGC 4698 (see Table 1), where there are no optical emission lines, would represent a recent or strong internal burst or external interaction which removed even the usual gaseous, optical emission regions leaving only the core of absorption line stars. This model also gives a possible evolutionary connection between recent observations of HII and other narrow line galaxies and the ejection of quasars. Several high or medium redshift emission line objects apparently emerging from low redshift active galaxies either in or entraining luminous filament/arms are given in the literature: NGC 4151 (Arp 1977, 2003), NGC 1232 (Arp 1982, 2003), NGC 7603 (López-Corredoira \& Gutiérrez 2002, 2004), NEQ3 (Arp 1977; Gutiérrez \& López-Corredoira 2004), NGC 1365 (Roy \& Walsch 1997), NGC 3628 (Arp et al. 2002), 53W-003 (Arp 1999), NGC 2639 (Arp et al. A\&A, submitted), etc. Many of these systems are known to have ULXs. Obviously deeper multi-color images and Chandra X-ray observations should yield important results on all of these objects ${ }^{4}$.

Another suggested explanation for these association of galaxies and quasars with different redshift is mesolensing by King objects. In López-Corredoira \& Gutiérrez (2004) this is discussed in more detail.

Acknowledgements. Thanks are given to the referee M. W. Pakull for useful comments and in particular criticisms on the observational bias.

\footnotetext{
${ }^{4}$ Unfortunately an application to make an observation of the two HII galaxies in the bridge between NGC 7603 and NGC 7603 B with Chandra was turned down.
} 


\section{References}

Angelini, L., Loewenstein, M., Mushotzky, R. F., et al. 2001, ApJ, 557, L35

Arp, H. 1977, ApJ, 218, 72

Arp, H. 1982, ApJ, 263, 54

Arp, H. 1987, Quasars, Redshifts and Controversies (Berkeley: Interstellar Media)

Arp, H. 1997, A\&A, 319, 33

Arp, H. C. 1998, Seeing Red (Montreal: Apeiron)

Arp, H. 1999, ApJ, 525, 594

Arp, H. 2001, ApJ, 549, 802

Arp, H. 2002, ApJ, 571, 615

Arp, H. 2003, A Catalogue of discordant Redshift Associations (Montreal: Apeiron), in press

Arp, H., \& Sulentic, J. 1979, ApJ, 229, 496

Arp, H., Burbidge, E. M., Chu, Y., et al. 2002, A\&A, 391, 833

Bradt, H. V. D., \& McClintock, J. E. 1983, ARA\&A, 21, 13

Buote, D., \& Canizares, C. 1996, ApJ, 468, 184

Burbidge, E. M. 1995, A\&A, 298, L1

Burbidge, E. M., Junkkarinen, V., \& Koski, A. 1979, ApJ, 233, 97

Burbidge, E. M., Burbidge, G. R., \& Arp, H. C. 2003, A\&A, 400, L17

Clements, D., McDowell, J. C., Shaked, S., et al. 2002, ApJ, 581, 974

Colbert, E., \& Ptak, A. 2002, ApJS, 143, 25

de Ruiter, H., Arp, H., \& Willis, A. 1977, A\&AS, 28, 211

Foschini, L., Di Cocco, G., Ho, L. C., et al. 2002a, A\&A, 392, 817

Foschini, L., et al. 2002b [arXiv: astro-ph/0209098]

Foschini, L., Ho, L., Masetti, N., et al. 2002c, A\&A, 396, 787

Freedman, W., Madore, B., Gibson, B., et al. 2001, ApJ, 553, 47

Gao, Y., Wang, Q. D., Appleton, P. N., \& Lucas, R. A. 2003, ApJ, 596, L171

Gutiérrez, C. M., \& López-Corredoira, M. 2004, ApJL, accepted [arXiv:astro-ph/0401596]
Humphrey, P., Fabbiano, G., Elvis, M., Church, M., \& Balucinska-Church, M. 2003, MNRAS, 344, 134

King, A. R., Davies, M. B., Ward, M. J., Fabbiano, G., \& Elvis, M. 2001, ApJ, 552, L109

Körding, E., Falcke, H., \& Markoff, S. 2002, A\&A, 382, L13

La Franca, F., Fiore, F., Vignali, C., et al. 2002, ApJ, 570, 100

López-Corredoira, M., \& Gutiérrez, C. M. 2002, A\&A, 390, L15

López-Corredoira, M., \& Gutiérrez, C. M. 2004, A\&A, in press [arXiv: astro-ph/0401147]

Maccacaro, T., Gioia, I. M., Wolter, A., Zamorani, G., \& Stocke, J. T. 1988, ApJ, 326, 680

Masetti, N., Foschini, L., Ho, L. C., et al. 2003, ApJ, 406, L27

Matsumoto, H., Tsuru, T., \& Koyama, K. 2001, ApJ, 547, L25

Mirioni, L. 2003, Ph.D. Thesis, University of Strasbourgh

Nowak, M., Smith, B., Donahue, M., \& Stocke, J. 2003, AAS, Head Meeting, 35, 13.16

Pakull, M. W., \& Mirioni, L. 2002 [arXiv: astro-ph/0202488]

Radecke, H.-D. 1997, A\&A, 319, 33

Pietsch, W., Vogler, A., Kahabka, P., Jain, A., \& Klein, U. 1994, A\&A, 284, 386

Roberts, T. P., Goad, M. R., \& Warwick, R. S. 2003, MNRAS, 342, 709

Roy, J.-R., \& Walsh, J. 1997, MNRAS, 288, 715

Strickland, D., Colbert, E., Heckman, T., et al. 2001, ApJ, 560, 707

Supper, R., Hasinger, G., Pietsch, W., et al. 1997, A\&A, 317, 328

Terashima, Y., \& Wilson, A. 2003, ApJ, 583, 145

Trinchieri, G., Sulentic, J., Breitschwerdt, D., \& Pietsch, W. 2003, A\&A, 402, 73

Véron-Cetty, M. P., \& Véron, P. 2003, A\&A, 412, 399

Vogler, A., \& Pietsch, W. 1999, A\&A, 352, 64

Wang, Q. D. 2002, MNRAS, 332, 764 